\title{
Assessment of the Complications and Associated Findings by Conventional MRI in Post-Operative ACL Reconstruction
}

\author{
Amina Shaker Ahmed*, Amal M. Hassan, Enass M. Khattab, Muhammed M. Elfawal \\ Radiodiagnosis Department, Faculty of Medicine, Zagazig University, Egypt \\ *Corresponding Author: Amina Shaker Ahmed, Email: Dr.aminashaker@yahoo.com
}

\begin{abstract}
Background: The anterior cruciate ligament (ACL) runs from the femur to the tibia in the knee joint and is a thick band of connective tissue that may be exposed to tears. Accurate MR imaging evaluation of these patients requires an understanding of the usual postoperative MR imaging appearance of postoperative ACL graft and any abnormalities.
\end{abstract}

Objective: To assess the complications and associated findings by conventional MRI in post-operative ACL reconstruction.

Patients and methods: This study included 27 symptomatic patients (23 males and 4 females). The study was carried out at the Radiology Department at Zagazig University Hospitals. They were assessed by conventional MRI after the ACL operations.

Results: The mean age of the studied patients was $29.26 \pm 7.79$ years old. Most of the studied patients were males $(85.2 \%) .14 .8 \%$ of the studied patients had anterior tibial translation, graft impingement in $2 / 27(7.4 \%)$ of patients and anteriorly displaced tibial hardware was found in $3 / 27(11.1 \%)$ of the studied patients. $18.5 \%$ of the studied patients had joint effusion. PCL buckling and bone marrow edema were found in $7.4 \%$ of the studied patients and PHMM degeneration was found in $11.1 \%$ of the studied patients.

Conclusion: In order to properly evaluate the post-operative knee, particularly in patients who had cruciate ligament repair, magnetic resonance imaging (MRI) of the knee is required.

Keywords: ACL reconstruction, Conventional MRI.

\section{INTRODUCTION}

Anterior cruciate ligament reconstruction is one of the most common orthopedic procedures performed worldwide $^{(\mathbf{1})}$.

The incidence of anterior cruciate ligament injuries especially in athletes has rapidly increased over the last decades. Rupture of ACL compromises the stability of the knee in individuals who are active, resulting in chronic instability and recurrent episodes of giving way. This injury presents a challenge for surgeons. The outcome is better in patients treated by surgical reconstruction of ACL than those managed conservatively ${ }^{(2)}$. The most common procedure is anterior cruciate ligament reconstruction ${ }^{(3)}$. In addition to an increase in the number of patients undergoing ACL surgery, an increase in the number of patients who require imaging following surgery because of failure to improve, recurrent symptoms or new damage is a result of this increase in ACL surgery ${ }^{(3)}$.

Post-operatively, MRI is useful because it can identify a variety of characteristics and scenarios that could indicate prospective difficulties to clinicians in a non-invasive method. Surgical results could be compromised, and failure of the ACL reconstruction could be predicted if graft signal and integrity, accurate tunnel placement, tunnel widening, and fixation device or donor site difficulties are present. The worst outcomes or a higher failure probability are connected with a number of knee anatomical characteristics. This article serves as a practical guide for the doctor who wishes to use MRI to assess the patient's recovery from ACL surgery, examine for normal or pathologic conditions by analysis of all the characteristics and traits directly or indirectly associated with ACL reconstruction ${ }^{(\mathbf{1})}$.

T2-weighted MR scans reveal ACL graft disruption, including the absence of complete graft fibers and an increase in signal intensity similar to that of fluid at the graft's expected location ${ }^{(4)}$.

Intact fibers remain in the graft despite partial tears, which show up as areas of elevated signal intensity in the graft ${ }^{(4)}$. Accurate MR imaging evaluation of patients who had an ACL transplant requires an understanding of the typical postoperative MR imaging appearance and abnormalities ${ }^{(4)}$.

\section{PATIENTS AND METHODS}

The research for this topic was done in the Radiology Department, Faculty of Medicine, Zagazig University Hospitals, after obtaining institutional review board approval and informed consent from patients or relatives before study, including 27 patients.

Inclusion criteria: Patient who underwent anterior cruciate ligament reconstruction more than six months at time of examination.

Exclusion criteria: Patient with contraindication for MRI examination (Patients with implanted pacemaker and other cardiological devices incompatible with MRI, patients with ocular implants and patients with aneurysmal clips) and arthritis.

All patients meeting the inclusion criteria were subjected to:

Demographic data including patients' name, age, sex and occupation. 
Clinical examination: Patient were assessed by the outpatient physician then redirected to the radiology unit.

Magnetic resonance imaging: The target knee was imaged using $1.5 \mathrm{~T}$. scanner (Achieva, PHILIPS Medical Systems) using the following sequences: Axial PD FAT SAT, coronal T2 weighted image, coronal PD FAT SAT, sagittal T2-weighted image and PD and sagittal T1-weighted image. A cylindrical phased-array extremity coil was used. The total time of examination is around $15 \mathrm{~min}$.

Table (1): Different parameters of pulse sequences

\begin{tabular}{||l|c|c|c||}
\hline Sequence tr & te & $\begin{array}{c}\text { Echo train } \\
\text { length }\end{array}$ \\
\hline 1-Axial T1 localizer & 400 & 20 & 4 \\
\hline 2-Axial T1 & $600-700$ & $17-20$ & 4 \\
\hline 3-Coronal fat \\
saturated PD & $\begin{array}{c}1800- \\
2000\end{array}$ & $17-20$ & 4 \\
\hline 4-Sagittal PD & $2000-$ & $17-20$ & \\
\hline 5-Sagittal T2 & 4000 & 100 & 4 \\
\hline
\end{tabular}

The scanning time for each sequence was between 2 and 3.5 minutes.

Table (2): Scanning parameters:

\begin{tabular}{||l||}
\hline Region of interest : Target knee \\
\hline Matrix: $256 \times 192$ \\
\hline FOV: $14 \mathrm{~cm}$ \\
\hline Collimation: \\
\hline Slice thickness: $3 \mathrm{~mm}$ \\
\hline Inter slice gap: $1.5 \mathrm{~mm}(50 \%$ overlap $)$ \\
\hline
\end{tabular}

\section{Patient' preparation:}

Good explanation of the procedure, asking for any implanted medical devices and asking about pregnancy. The MRI machine is a tight, enclosed space. If the patient was claustrophobic, He was given the option to take a sedative or withdrawn from the test. Removal of any non-fixed metal prosthesis, that might interfere with scanning of the region of interest. Asking the patient to be calm, take quiet breathing and instruct him/her not to move during the procedure.

Patient Position: Supine position, head first towards the gantry.

\section{Scanning Instructions:}

Table position: Setting the table height so that the area to be scanned (target knee joint) is centered in the scan field. No change of table position between images so that all images create one unified volume.

Image acquisition: Examination and interpretation of the images was done under the guidance of qualified musculoskeletal radiologists on a workstation, (PHILIPS Intelli Space Portal) and the picture archiving and communication system (PACS).

\section{Ethical consent:}

An approval of the study was obtained from Zagazig University Academic and Ethical Committee. Every patient signed an informed written consent for acceptance of the study. This work has been carried out in accordance with The Code of Ethics of the World Medical Association (Declaration of Helsinki) for studies involving humans.

\section{Statistical Analysis:}

To analyse the collected data, we used the Statistical Package for Social Services version 24 (SPSS), which produced tables and graphs that represented the findings. Categorical qualitative variables were expressed as absolute frequencies (number) or relative frequencies (\%) and continuous quantitative statistics, like age, were expressed as mean \pm standard deviation, and median). When the significance probability was equal or less than 0.05 , the results were considered statistically significant (P 0.05). To be termed highly significant (HS), the P-value had to be less than or equal to 0.001 ; otherwise, it was deemed statistically insignificant (NS).

\section{RESULTS}

The mean age of the studied patients was $29.26 \pm 7.79$ years old, with a range from 20 to 43 years old and most of the studied patients were males (85.2\%) (Table 3). The most considerable complain found in all patient was knee pain (100\%) and knee pain was accompanied with limitation of movement in $25.9 \%$. Additionally, pain was accompanied with edema in only $3.7 \%$ (Table 4).

$14.8 \%$ of the studied patients had anterior tibial translation, graft impingement in $2 / 27(7.4 \%)$ of patients and anteriorly displaced tibial hardware was found in 3/27 (11.1\%) of the studied patients (Table 5).

$18.5 \%$ of the studied patients had joint effusion, PCL buckling and bone marrow edema were found in $7.4 \%$ of the studied patients and PHMM degeneration was found in $11.1 \%$ of the studied patients (Table 6).

Table (3): Demographic data of the studied patients

\begin{tabular}{||l|c|c|}
\hline \multirow{2}{*}{ Demographic data } & \multicolumn{2}{|c|}{$\begin{array}{c}\text { Studied patients } \\
(\mathbf{N}=27)\end{array}$} \\
\cline { 2 - 3 } & No. & \% \\
\hline Sex & 23 & 85.2 \\
\hline Male & 4 & 14.8 \\
\hline Female & \multicolumn{2}{|l|}{} \\
\hline Age (years) & $29.26 \pm 7.79$ \\
\hline Mean \pm SD & $27(20-43)$ \\
\hline Median (Range)
\end{tabular}


Table (4): Complain post-operative ACL reconstruction among the studied group

\begin{tabular}{||l|c|c||}
\hline \multirow{2}{*}{\multicolumn{1}{|c|}{ Item }} & \multicolumn{2}{c|}{ Studied gr (N=27) } \\
\cline { 2 - 3 } Complain & \multicolumn{2}{c|}{ No. } \\
\hline Knee pain & 27 & $100.0 \%$ \\
\hline Edema & 1 & $3.7 \%$ \\
\hline Limitation of movement & 7 & $25.9 \%$ \\
\hline
\end{tabular}

Table (5): post-operative ACL reconstruction complication among the studied patients

\begin{tabular}{||l|c|c|}
\hline \multicolumn{1}{|c|}{ Item } & \multicolumn{2}{c|}{ Studied patients (N=27) } \\
\cline { 2 - 3 } & No. & \% \\
\hline erior displacement of tibial tunnel & 3 & $11.1 \%$ \\
\hline erior tibial translation & 4 & $14.8 \%$ \\
\hline placement femoral screw & 1 & $3.7 \%$ \\
\hline Graft roof impingement & 2 & $7.4 \%$ \\
\hline Posteriorly displaced tibial hardware & 1 & $3.7 \%$ \\
\hline \hline
\end{tabular}

Table (6): Associated finding among the studied patients

\begin{tabular}{||l|c|c||}
\hline \multicolumn{1}{c|}{ Item } & \multicolumn{2}{c||}{ Studied patients(N=27) } \\
\cline { 2 - 3 } & No. & \% \\
\hline Joint effusion & 5 & $18.5 \%$ \\
\hline Sublaxation of patella & 1 & $3.7 \%$ \\
\hline Bone marrow edema & 2 & $7.4 \%$ \\
\hline PCL buckling & 2 & $7.4 \%$ \\
\hline Intercondylar edema & 1 & $3.7 \%$ \\
\hline Torn PHMM & 1 & $3.7 \%$ \\
\hline Degeneration of PHMM & 3 & $11.1 \%$ \\
\hline \hline
\end{tabular}

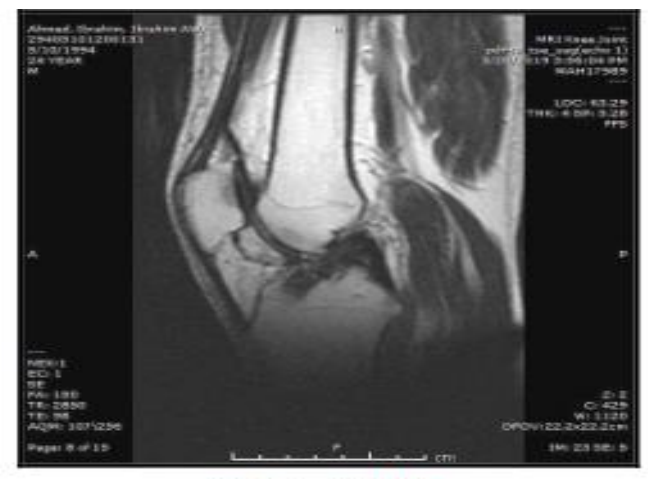

(A): Sagittal T2:

High SI and thickening of ACL fibers contacting the intercondyler roof.

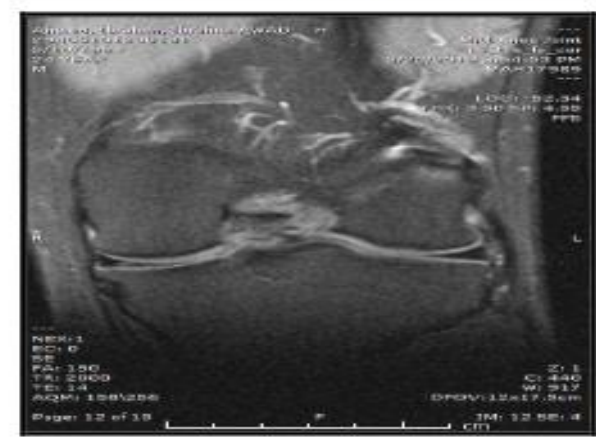

(B) Coronal Stir:

Preserved femoral attachment of ACL graft.

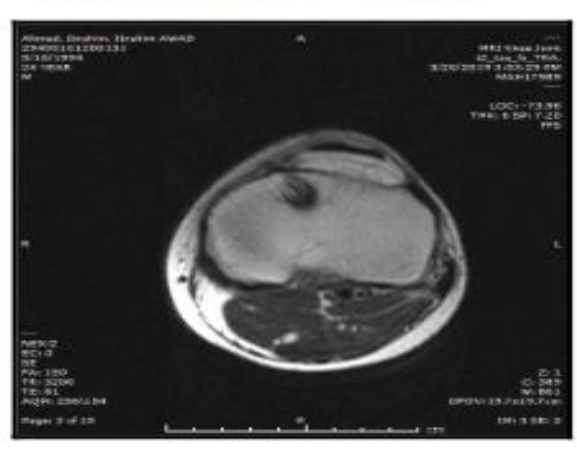

(C) Axial T2:

Anterior location of tibial tunnel.

Figure (1): Sagittal T2 (A) \& Coronal Stir (B) \& Axial T1 (C): Relative anterior location of tibial tunnel and subsequent high SI and thickening of ACL fibers contacting the intercondyler roof denoting impingement and partial tear. Mild synovial effusion. Diagnosis was graft partial tear and impingement. 


\section{DISCUSSION}

After surgery, MRI is crucial for postoperative knee evaluation, especially for symptomatic anterior cruciate ligament reconstruction knee evaluation. By using MRI, the anterior cruciate ligament graft, bone tunnels, and any other knee problems can be examined with precision. If the graft fails, MRI can show the location of the bone tunnel, impingement, and arthrofibrosis (11). The findings of our study revealed that the mean age of the examined patients is $29.26 \pm$ 7.79 years old, with a range from 20 to 43 years old, and that the majority of the studied patients were males (85.2 percent). Collins et al. ${ }^{(5)}$ published a study in which over 2,370 patients in Boston were found to have an ACL injury between 2001 and 2007. A grand total $47 \%$ of the participants were females, while $53 \%$ were males. In our research, $85.2 \%$ of participants were men, whereas $14.8 \%$ were women. In Collins et al. ${ }^{(5)}$ study, the average age was 47.1 ( 29 in our study). In our study mean age at diagnosis being 29 years old (Range: $20-$ 43 years), which is in concordance with Farahat $\boldsymbol{e t}$ al. (13) where mean age was 32 years, which could be explained by the higher level of activity among western population above 40 years and their higher life expectancy compared to our locality.

At the time of presentation, all 27 patients in our study had symptoms, and the most common complaint was knee pain (100 percent of the time), which was accompanied by movement limitation in 25.9 percent of cases and pain with edema in just 3.7 percent. Galal $\boldsymbol{e t}$ al. ${ }^{(6)}$ examined patient symptoms in 2011. Publicly available information on 42 patients who presented with the symptoms listed below when diagnosed 100 percent of people reported pain, 75 percent reported unstable locking, 52 percent reported swelling, 29.16 percent reported clicking, and $20.8 \%$ reported failure to restore full extension.

The intra-articular component of the graft showed as a thick band of uniformly low signal intensity that extends parallel and posterior to the intercondylar notch on sagittal MR images taken immediately after surgery (13). There was a varying amount of enhanced signal across intact fibers on all MR imaging sequences in the first 1-3 months following ACL reconstruction because of revascularization and an increase in body water content ${ }^{(7)}$.

The current study revealed that $14.8 \%$ of the studied patients had anterior tibial translation, graft impingement in $2 / 27(7.4 \%)$ of patients and anteriorly displaced tibial hardware was found in 3/27 (11.1\%) of the studied patients. $18.5 \%$ of the studied patients had joint effusion, PCL buckling and bone marrow edema were found in $7.4 \%$ of the studied patients and PHMM degeneration was found in $11.1 \%$ of the studied patients. In Murakami $\boldsymbol{e t}$ al. ${ }^{(8)}$ study, a high signal intensity tissue was identified to surround the graft 1-3 months after surgery. By 7-12 months, this strong signal had spread throughout the graft and surrounded the individual bundles. Because of this, after 19 months after surgery the graft was visible as a low signal intensity band that was either homogeneous or had minor hyperintensities surrounding the individual fibers of the graft. Several primary and secondary symptoms point to ACL injuries being present. Main indicators are absence or discontinuity of ACL and morphologic and signal intensity abnormalities such as the retraction or fibrilization of PCL in remote tears ${ }^{(9)}$. The anterior drawer sign, a buckling PCL, an exposed PHLM, and bone contusions in a specific region are all secondary indicators of an ACL tear ${ }^{(10)}$.

\section{CONCLUSION}

MRI knee imaging is critical in post-operative evaluations, especially for patients who had cruciate ligament reconstruction surgery. Anterior cruciate ligament graft reconstruction surgery that fails can be evaluated with MR imaging. MR imaging can accurately detect anterior cruciate ligament graft problems including aberrant tunnel alignment, roof impingement, partial and total graft tears, hardware loosening, and infection.

Financial support and sponsorship: Nil.

Conflict of interest: Nil.

\section{REFERENCES}

1. Grassi A, Bailey J, Signorelli C et al. (2016): Magnetic resonance imaging after anterior cruciate ligament reconstruction: A practical guide. World J Orthop., 7 (10): 638- 649.

2. Radwan M, Mostafa M, AbdElaziz T (2014): Anterior cruciate ligament reconstruction using hamstring tendon autograft in young football players. Egyptian Orthopedic Journal, 14: 60-65.

3. Recht M, Kramer J (2002): MR imaging of postoperative knee: a pictorial essay. Radiographic, 174: 765-74.

4. Trattnig S, Rand T, Czerny C et al. (1999): Magnetic resonance imaging of the postoperative knee. Top Magn Reson Imaging, 10: 221 236

5. Collins JE, Katz JN, Donnell-Fink LA, Martin SD and Losina E. (2013): Cumulative incidence of ACL reconstruction after ACL injury in adults: Role of age, sex and race. Am J Sports Med.. 41 (3): 544-9.

6. Galal A, Abdul-Maksoud S, Abdul-Salam S et al. (2011): Anterior cruciate ligament reconstruction: Magnetic resonance imaging and factors influencing outcome. The Egyptian Journal of Radiology and Nuclear Medicine, 42: 193- 200.

7. Johnson D, Houghton T, Radford P (1986): Anterior midline or medial parapatellar incision for arthroplasty of the knee. A comparative study. J Bone Joint Surg., 68: 812-18.

8. Murakami Y, Sumen Y, Ochi M et al. (1999): Appearance of anterior cruciate ligament autografts in their tibial bone tunnels on oblique axial MRI. Magn Reson Imaging, 17: 679-687.

9. Mink J, Levy T, Crues J (1988): Tears of the anterior cruciate ligament and menisci of the knee: MR imaging evaluation. Radiology, 167: 769-74.

10. Vahey T, Hunt J, Shelburne K (1993): Anterior translocation of the tibia at MR imaging: a secondary sign of anterior cruciate ligament tear. Radiology, 22:411-16.

11. Weber W, Neumann C, Barakos J et al. (1991): Lateral tibial rim (segond) fractures MR imaging characteristics. Radiology, 180: 731-4.

12. Farahat A, Eid M, El-Khadrawe T et al. (2015): MRI findings in symptomatic patients following anterior cruciate ligament surgery. The Egyptian Journal of Radiology and Nuclear Medicine, 46: 405-411.

13. Tomczak R, Hehl G, Mergo $P$ et al. (1997): Tunnel placement in anterior cruciate ligament reconstruction: MRI analysis as an important factor in the radiological report. Skeletal Radiol., 26: 409-13. 